\title{
Ni-Mo Alloys with a High Molybdenum Content Using Pulse-Plating Method
}

\author{
Seichi REnGakuJi, Takakazu TAKANO ${ }^{\dagger}$, Yuuko NAKAMURA, Susumu IKENo \\ and Choichiro SHIMASAKI
}

Received December 22, 1997 ; Accepted April 20, 1998

\section{INTRODUCTION}

The electrodeposition mechanism of Ni-Mo alloys has been classified as induced codeposition by Brenner. ${ }^{1)}$ One of problems in this codeposition is that the amount of molybdenum in the alloy is limited by the catalytic ability of the iron group metals. The maximum molybdenum content in the metallic $\mathrm{Ni}-\mathrm{Mo}$ alloy has been believed to be about 20 $w t \% .^{2-5)}$ Though alloys with higher molybdenum content were desirable for their wear and corrosion resistance, the alloy that exceeded this value was nonmetallic and contained certain amount of oxygen. ${ }^{6)}$ Recently, Podlaha et al. ${ }^{7,8)}$ found that bright, adherent, and metallic Ni-Mo alloys containing over 48 wt $\%$ molybdenum were electrodeposited from an aqueous solution using a rotating cylinder electrode. This experimental result suggests that the mass transport of ion species in the electrolytes appears to play an important role in the induced deposition. However, the rotating cylinder electrode technique is not applied for planer products which is widely used for industry. By using the pulse-plating technique, an alloy with a high molybdenum content may be obtained since the pulse-plating technique is efficient to affect mass transport of ions. Actually metallic alloy containing $25 \mathrm{wt} \%$ molybdenum was

Department of System Engineering of Materials and Life Science, Faculty of Engineering, Toyama University (Gofuku, 3190, Toyama 9308555, Japan)

†NACHI Fujikoshi corp., (Ishigane, 20, Toyama 930-8511, Japan)

Key Words : Electroplating, Ni-Mo Alloys obtained by pulsed plating from an acidic citrate bath $^{9}$. In this paper, it is shown that metallic Ni-Mo alloys containing $38 \mathrm{wt} \%$ molybdenum, which exceed previous maximum molybdenum contents of $25 \mathrm{wt} \%$, are obtained by pulsed plating from an acidic citrate bath at lower duty cycle.

\section{EXPERIMENTAL}

A plating bath containing $\mathrm{n}(\mathrm{n}=0.01,0.05,0.1$, $0.2) \mathrm{M}$ sodium molybdate $-0.36 \mathrm{M}$ nickel sulfate - 0.3 M sodium citrate were prepared from reagent grade chemicals (Kanto Chem. Co.) and doubly distilled water. The bath was kept at 25 ${ }^{\circ} \mathrm{C}$ and adjusted to $\mathrm{pH} 3.5$ with conc. sulfuric acid. Using a parallel and planar electrode configuration with a platinum anode and a copper cathode, each $20 \times 40 \mathrm{~mm}$ in size, the plating of the Ni-Mo alloys was carried out without any stirring of the bath. Rectangular pulse plating was performed using a bipolar power supply (Kikusui PBX 40-2.5). Various peak current densities, pulse frequencies, and duty cycles were employed. The composition of the electrodeposits about $1 \mu \mathrm{m}$ in thickness was determined by wavelength dispersive $\mathrm{X}$ ray spectroscopy (Shimazu Co., EPMA-1500).

\section{RESULTS AND DISCUSSION}

The relations between the concentration of molybdate ion in the bath and the molybdenum content in alloys electrodeposited at various direct or pulsed current densities, $i_{c}$, are shown in Fig. 1. Pulsed plating was performed using a duty cycle of 0.5 and pulse frequency of 100 Hz. In both cases, the molybdenum content increased with an increase in molybdate ion concentration. But, beyond the $0.1 \mathrm{M}$ molybdate 


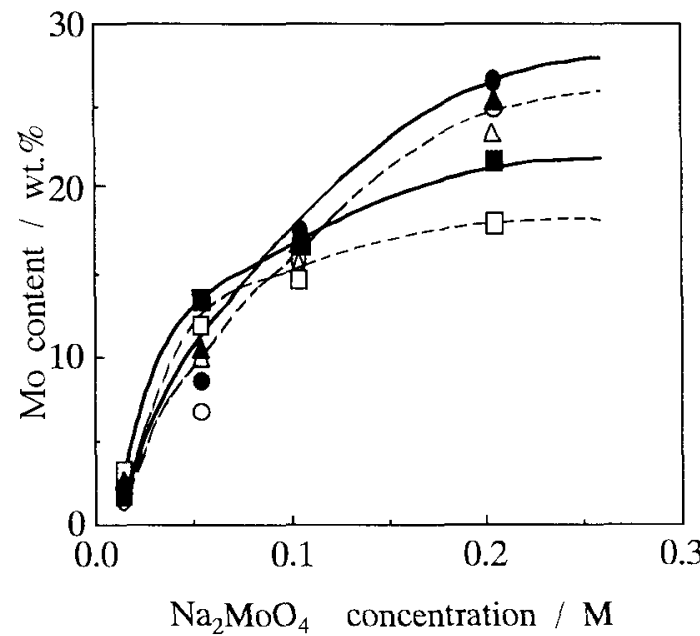

Fig. 1 The changes in molybdenum content with molybdate ion concentration at $5 \mathrm{Adm}^{-2}(0), 10 \mathrm{Adm}^{-2}$

( $\mathbf{A}), 20 \mathrm{Adm}^{-2}(\mathbf{D})$, pulsed $\mathrm{i}_{\mathrm{c}}$ under pulse frequency of $100 \mathrm{~Hz}$ and duty cycle of 0.5 and at $5 \mathrm{Adm}^{-2}(\mathrm{O}), 10$ $\operatorname{Adm}^{-2}(\triangle)$ and $20 \mathrm{Adm}^{-2}(\square)$ of direct $i_{c}$.

ion concentration, the molybdenum content decreased with an increase in $i_{c}$. This experimental result arises from induced deposition mechanism. Molybdenum content is determined by both the catalytic ability of hydrogen attached on freshly deposited nickel and the amount of intermediate molybdenum oxide reduced from molybdate ion. More over, it had been clarified that the molybdenum content increased with increasing concentration of molybdate ion in the bath and decreased with increasing direct cathodic current density ${ }^{4}$. This experimental result suggests that alloy formation through the reduction of intermediate molybdenum oxide to metallic state is depressed at high current density and high molybdate ion concentration, and so the increment tendency of molybdenum content with molybdate ion concentration changes with the cathodic current density and molybdate ion concentration.

In addition, the molybdenum content obtained from a pulsed current (solid lines in Fig.1) is somewhat greater than that from a direct current (dotted lines in Fig.1). These increments of molybdenum content might be due to the extra delivery of molybdate ion during the off-time. We did not employ the average current density which usually used to pulse method, because

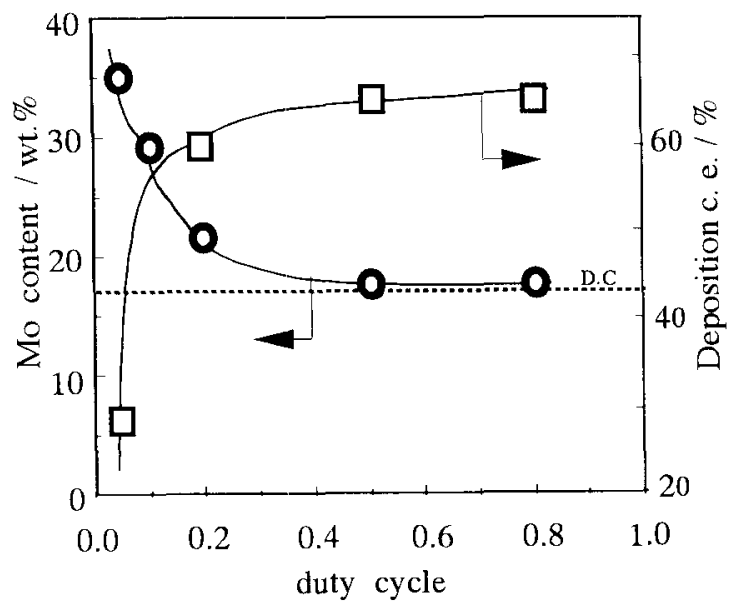

Fig. 2 The changes in Mo content $(\bigcirc)$ and deposition current efficiency $(\square)$ with duty cycles at the peak $i_{c}$ of $5 \mathrm{Adm}^{-2}$. The dotted line represents the Mo content at the direct $\mathrm{i}_{\mathrm{c}}$ of $5 \mathrm{Adm}^{-2}$. The concentration of sodium molybdate in the bath is $0.1 \mathrm{M}$.

composition of deposits is controlled by applied current density.

To clarify the effect of off-time on the molybdenum content, pulse plating was carried out in the following way, that is, the interval during the current flow in each pulse cycle, the so-called on-time, was fixed at $5 \mathrm{~ms}$ and the off-time was varied from 1.25 to $95 \mathrm{~ms}$. This variation corresponded to changes in the duty cycles from 0.8 to 0.05 . The changes in molybdenum content and deposition current efficiency with duty cycles at the constant peak $i_{c}$ of $5.0 \mathrm{Adm}^{-2}$ are shown in Fig. 2. The dotted line in Fig. 2 represents the molybdenum content in alloys electrodeposited at the direct $i_{c}$ of $5.0 \mathrm{Adm}^{-2}$. The current efficiency was $52 \%$ at the same direct $i_{c}$. It is clear that the molybdenum content depends on the duty cycles, and is greater than that from a direct current. Metallic Ni-Mo alloys containing over $25 \mathrm{wt} \%$ molybdenum are obtained at a duty cycle lower than 0.2 and its content significantly exceeded the previous maximum value ${ }^{9}$. When the alloy deposited from the duty cycle of 0.05 , it contained $38 \mathrm{wt} \%$ molybdenum, though a nickel rich phase was partially observed. Now, we are seeking for optimum plating conditions to get uniform, bright, and metallic 
Ni-Mo alloys with high molybdenum content.

\section{References}

1)Brenner, "Electrodeposition of Alloys" Vol.II. (1963) Academic press, New York \& London, p399-408.

2)D.W.Ernst and M.L.Holt, J.Electrochem.Soc., 105, 686 (1958).

3)S.Rengakuji, Y.Nakamura, K.Nishibe, M.Inoue, and T.Komura, Denki Kagaku, 62, 602 (1994).

4)S.Rengakuji, Y.Nakamura, N.Sumi, K.Nishibe, and T.Komura, Denki Kagaku,
63,400 (1995).

5)E.Chassaing, K.Vu Quang, and R.Wiart, J.Appl.Electrochem., 19, 839(1989).

6)S.Rengakuji, K.Nishibe, Y.Nakamura, and H.Imanaga, Denki Kagaku, 58, 63 (1990).

7)E.J.Podlaha, M.Matlosz, and D.Landolt, J.Electrochem.Soc., 140, L149(1993).

8)E.J.Podlaha and D.Landolt, J. Electrochem. Soc., 143, 885(1996).

9)C.C.Nee, W.Kim, and R.Weil, J.Electrochem. Soc., 135, 1100(1988). 